\title{
Joint Source-Channel Decoding over MIMOChannels Based on Partial Marginalization
}

\author{
Daniel Persson, Erik G. Larsson and Mikael Skoglund
}

\section{Linköping University Post Print}

N.B.: When citing this work, cite the original article.

(C)2012 IEEE. Personal use of this material is permitted. However, permission to reprint/republish this material for advertising or promotional purposes or for creating new collective works for resale or redistribution to servers or lists, or to reuse any copyrighted component of this work in other works must be obtained from the IEEE.

Daniel Persson, Erik G. Larsson and Mikael Skoglund, Joint Source-Channel Decoding over MIMOChannels Based on Partial Marginalization, 2012, IEEE Transactions on Signal Processing, (60), 12, 6734-6739. http://dx.doi.org/10.1109/TSP.2012.2214215

Postprint available at: Linköping University Electronic Press http://urn.kb.se/resolve?urn=urn:nbn:se:liu:diva- 86182 


\title{
Joint Source-Channel Decoding over MIMO Channels Based on Partial Marginalization
}

\author{
Daniel Persson, Erik G. Larsson, and Mikael Skoglund
}

\begin{abstract}
We investigate fast joint source-channel decoding employed for communication over frequency-flat and frequencyselective block-fading multiple-input multiple-output channels. Our setting has applications for communication with short codes under low-latency constraints. The case of no transmitter channel state information is considered.

We propose a partial marginalization decoder that allows performance to be traded for computational complexity, by adjusting a user parameter. By tuning this parameter to its maximum value, the minimum mean square error (MMSE) decoder is obtained. In the conducted simulations, the proposed scheme almost achieves the MMSE performance for a wide range of the channel signal-to-noise ratios, with significant reductions in computational complexity.
\end{abstract}

Index Terms-Joint source-channel coding, multiple-input multiple-output (MIMO), short codes, low latency, fast decoding.

\section{INTRODUCTION}

Multiple-input multiple-output (MIMO) technology improves both capacity and robustness in traditional communications [1]. While MIMO usually refers to a setting with many antennas, our proposed method is applicable to any channels with cross-talk. We consider joint source-channel coding and communication with short codes, which find applications for low delay transmissions. When a number of i.i.d. zero-mean Gaussian sources are mapped to as many i.i.d. zero-mean Gaussian channels without cross-talk, the distortion is measured in mean square error (MSE), and the decoder knows the source and channel noise variances, analog uncoded transmission is optimal [2]. However, in this treatment, we have general sources, different numbers of source and channel dimensions, and channel cross-talk. While many treatments of analog short codes exist, see, e.g., our schemes [3], [4], digital communication systems are cheaper to manufacture, and signal fidelity is easier to control [5]. We assume the transmission to be fully digital. In a scenario with short codes, the source-channel separation theorem in general does not apply, and the optimal solution consists of joint optimization

Copyright (c) 2012 IEEE. Personal use of this material is permitted. However, permission to use this material for any other purposes must be obtained from the IEEE by sending a request to pubs-permissions@ieee.org.

This work was supported in part by Security Link, the Swedish Research Council (VR), the Swedish Foundation for Strategic Research (SSF), and ELLIIT. E. G. Larsson is a Royal Swedish Academy of Sciences (KVA) Research Fellow supported by a grant from the Knut and Alice Wallenberg Foundation.

D. Persson and E. G. Larsson are with the Department of Electrical Engineering, Linköping University, SE-581 83 Linköping, Sweden (email: danielp@isy.liu.se, egl@isy.liu.se). M. Skoglund is with the School of Electrical Engineering, Royal Institute of Technology, SE-100 44 Stockholm, Sweden (email: skoglund@ee.kth.se). of a source and a channel code. Many treatments exist for the case of full channel state information (CSI) available at both the transmitter and receiver [6]-[9]. This paper focuses on the setting with frequency-flat and frequency-selective blockfading channels, where no CSI is available at the transmitter, while the receiver has full CSI. This situation arises when the receiver can estimate the channel from pilots, and where there are neither channel reciprocity nor any feedback channels. Our transmitter also does not consider the channel when quantizing the source vectors, and mapping the quantizer indices to channel codewords. It does not optimize the number of quantization vectors, and does not add redundant bits as a means for protecting the message. Our decoding scheme however utilizes knowledge of the probabilities for the different quantizer decisions.

The decoding method in this paper is of soft type [10], i.e., several source reconstruction vectors are weighted together given their probabilities of being chosen at the transmitter, and given the received channel vector. The MSE-optimal reconstruction has exponential computational complexity in the number of transmit antennas. Joint source-channel coding has not yet benefited from the large amount of research on partial marginalization (PM) and fixed complexity sphere decoder (FCSD) type MIMO detectors [11]-[14] proposed for MIMO communications with long codes. We propose an algorithm of PM type for approximate minimum mean square error (MMSE) decoding. A survey on previous work on the PM algorithm is given in Sec. I-A. We thereafter summarize the contribution of this paper in Sec. I-B.

\section{A. Background}

The PM detector [11] was proposed for approximate maximum a posteriori (MAP) MIMO demodulation for decoding of long codes such as low-density parity-check and turbo codes with soft output. The algorithm was later developed to work with general constellations and soft input [12], and it has been applied in a MIMO detection resource allocation problem, where different computational complexity levels are employed for the different channel realizations over which a long codeword spans [15]. Further, the distribution of the PM detection log-likelihood ratio (LLR) has been analyzed in the high channel signal-to-noise ratio (CSNR) regime in [16].

The approximation in the PM algorithm consists of two steps. In the first step, a carefully chosen set of marginalization sums is approximated by their largest terms. This operation has given the algorithm its name. In the second step, a lowcomplexity method of zero forcing with decision feedback 
(ZF-DF) type is used to find these largest terms approximately. The advantages of PM over the sphere decoder [17] are that it offers a constant and fully predictable runtime, and that it is straightforward to parallellize. PM trades performance for computational complexity via a user-defined parameter $r$ in a well-defined manner. When setting $r$ to its largest possible value, the algorithm becomes the maximum a posteriori demodulator, and by setting the parameter to zero, one obtains a ZF-DF-type solution. The difference between the PM and the FCSD [13], [14] is that FCSD uses an additional approximation, namely, the replacement of the remaining marginalization sums by a max-log approximation, but finding the maximum costs essentially as much as summing all terms in PM.

\section{B. Our contribution}

Our contribution is a PM-type algorithm for approximating the MMSE joint source-channel decoding of short codes. The presented PM algorithm differs from the algorithms in [11], [12] in two ways. A source vector estimate is now supplied instead of a bit estimate. We also show how we are able to deal with joint prior probabilities of the transmitted symbols.

Similarly to previously proposed PM algorithms, the proposed PM algorithm uses an algorithm of ZF-DF-type as a building block for the approximations in order to achieve a simple parallelization with a fully predictable runtime. We however comment on how to extend the proposed ZF-DF algorithm to a full sphere decoder which can deal with general joint prior probabilities. This generalized sphere decoder could be used for other problems involving joint probability maximization. In the conducted simulations, the proposed scheme almost achieves the MMSE performance for a wide range of the CSNR, with significant reductions in computational complexity. We discuss the choice of the parameter $r$, and make comparisons to rate-distortion-capacity performance limits.

\section{Partial Marginalization MiMO DeCODing of SHORT CODES}

In our setting with short codes, it is not optimal to design the source and channel coders separately. At the transmitter, we however do not have CSI, and we thus have to resort to separate source and channel coding. At the receiver however, joint decoding is applied, as will now be explained. The source vector $\boldsymbol{x} \in \mathbb{R}^{D}$ is to be quantized to an index $m=1, \ldots, M$. The index $m$ is transmitted by means of mapping $m$ to a set of indices $m_{k} \in\left\{1, \ldots, M_{1 \mathrm{D}}\right\}$, where $M_{1 \mathrm{D}}$ is the number of constellation points per transmit antenna, and where $m_{k}$ is sent on antenna $k$, for $k=1, \ldots, N_{\mathrm{T}}$, where $N_{\mathrm{T}}$ is the total number of transmit antennas. The corresponding modulation symbol vector is $\boldsymbol{s}=\left[s_{1}\left(m_{1}\right), \ldots, s_{N_{\mathrm{T}}}\left(m_{N_{\mathrm{T}}}\right)\right]^{T} \in \mathbb{R}^{N_{\mathrm{T}}}$, and $M=M_{1 \mathrm{D}}^{N_{\mathrm{T}}} .{ }^{1}$ Since the transmitter does not have CSI, the optimal mapping cannot be used. The following development

\footnotetext{
${ }^{1}$ We have chosen to work with a real-valued signaling constellation. However, it is straightforward to employ the decoding strategy with complexvalued signaling, even though no new fundamental aspects are added by such an extension.
}

does not assume any specific mapping of $m$ to $s$. The encoder centroids are defined as

$$
\mathrm{E}_{\boldsymbol{x} \mid m}[\boldsymbol{x}]=\int_{\boldsymbol{x} \in \Omega_{m}} \boldsymbol{x} p(\boldsymbol{x} \mid m) d \boldsymbol{x},
$$

where $\Omega_{m}$ is the source region corresponding to the index $m$, and $p(\boldsymbol{x} \mid m)$ is the probability distribution for $\boldsymbol{x}$ given that index $m$ was chosen. For $x \in \Omega_{m}$, the signal vector $s(m)$ representing index $m$ is transmitted over a MIMO channel

$$
\boldsymbol{y}=\boldsymbol{H} \boldsymbol{s}+\boldsymbol{n},
$$

where $\boldsymbol{y} \in \mathbb{R}^{N_{\mathrm{R}}}$ is the received vector, $\boldsymbol{H} \in \mathbb{R}^{N_{\mathrm{R}} \times N_{\mathrm{T}}}$ is the channel matrix, $\boldsymbol{n} \in \mathbb{R}^{N_{\mathrm{R}}}$ is the Gaussian channel noise vector with i.i.d. zero mean components with variance 1 , and $N_{\mathrm{R}}$ is the number of receive antennas. The MMSE receiver is

$$
\begin{aligned}
\mathrm{E}_{\boldsymbol{x} \mid \boldsymbol{y}}[\boldsymbol{x}] & =\int_{\boldsymbol{x}} \boldsymbol{x} p(\boldsymbol{x} \mid \boldsymbol{y}) d \boldsymbol{x} \\
& =\sum_{m=1}^{M} \int_{\boldsymbol{x}} \boldsymbol{x} p(\boldsymbol{x} \mid m, \boldsymbol{y}) p(m \mid \boldsymbol{y}) d \boldsymbol{x} \\
& =\sum_{m=1}^{M} \int_{\boldsymbol{x} \in \Omega_{m}} \boldsymbol{x} p(\boldsymbol{x} \mid m) p(m \mid \boldsymbol{y}) d \boldsymbol{x} \\
& =\frac{\sum_{m=1}^{M} \mathrm{E}_{\boldsymbol{x} \mid m}[\boldsymbol{x}] p(\boldsymbol{y} \mid m) p(m)}{\sum_{m=1}^{M} p(\boldsymbol{y} \mid m) p(m)},
\end{aligned}
$$

where $p(\boldsymbol{y} \mid m)$ is the probability of receiving $\boldsymbol{y}$ when index $m$ was sent, and $p(m)$ is the probability that index $m$ is chosen by the quantizer. Equation (3) is given by definition, (4) by the law of total probability, (5) by observing that $p(\boldsymbol{x} \mid m, \boldsymbol{y})=p(\boldsymbol{x} \mid m)$ and that $p(\boldsymbol{x} \mid m)=0$ if $\boldsymbol{x} \notin \Omega_{m}$. Finally, (6) is given by Bayes' rule and the law of total probability.

We will now develop an approximation of PM type to the MMSE receiver in (6). The first step is to rewrite (6) as

$$
\begin{aligned}
& \mathrm{E}_{\boldsymbol{x} \mid \boldsymbol{y}}[\boldsymbol{x}] \\
& =\frac{\sum_{m_{1}=1}^{M_{1 \mathrm{D}}} \ldots \sum_{m_{N_{\mathrm{T}}}}^{M_{1 \mathrm{D}}} \mathrm{E}_{\boldsymbol{x} \mid m_{1}, \ldots, m_{N_{\mathrm{T}}}}[\boldsymbol{x}] a\left(m_{1}, \ldots, m_{N_{\mathrm{T}}}\right)}{\sum_{m_{1}=1}^{M_{1 \mathrm{D}}} \ldots \sum_{m_{N_{\mathrm{T}}}=1}^{M_{1 \mathrm{D}}} a\left(m_{1}, \ldots, m_{N_{\mathrm{T}}}\right)},
\end{aligned}
$$

where

$$
a\left(m_{1}, \ldots, m_{N_{\mathrm{T}}}\right)=p\left(\boldsymbol{y} \mid m_{1}, \ldots, m_{N_{\mathrm{T}}}\right) p\left(m_{1}, \ldots, m_{N_{\mathrm{T}}}\right) .
$$

The PM scheme is based on two approximations. The crucial parameter here is the integer $r$, which trades off between computational complexity and MMSE performance. The first approximation consists of replacing $N_{\mathrm{T}}-r$ of the sums in (7) by the term that corresponds to the maximum of (8) for a given $\boldsymbol{y}$ and given $m_{N_{\mathrm{T}}-r+1}, \ldots, m_{N_{\mathrm{T}}}$

$$
\hat{m}_{1}, \ldots, \hat{m}_{N_{\mathrm{T}}-r}=\underset{m_{1}, \ldots, m_{N_{\mathrm{T}}-r}}{\arg \max } a\left(m_{1}, \ldots, m_{N_{\mathrm{T}}}\right) .
$$

We arrive at (10), which is shown on top of the next page. The second PM approximation consists of approximating the maximization in (9) by means of a ZF-DF-type scheme. We prepare for performing the ZF-DF algorithm by rewriting (9) 


$$
\mathrm{E}_{\boldsymbol{x} \mid \boldsymbol{y}}[\boldsymbol{x}] \approx \frac{\sum_{m_{N_{\mathrm{T}}-r+1}=1}^{M_{1 \mathrm{D}}} \ldots \sum_{m_{N_{\mathrm{T}}}=1}^{M_{1 \mathrm{D}}} \mathrm{E}_{\boldsymbol{x} \mid \hat{m}_{1}, \ldots, \hat{m}_{N_{\mathrm{T}}-r}, m_{N_{\mathrm{T}}-r+1}, \ldots, m_{N_{\mathrm{T}}}}[\boldsymbol{x}] a\left(\hat{m}_{1}, \ldots, \hat{m}_{N_{\mathrm{T}}-r}, m_{N_{\mathrm{T}}-r+1}, \ldots, m_{N_{\mathrm{T}}}\right)}{\sum_{m_{N_{\mathrm{T}}-r+1}=1}^{M_{1 \mathrm{D}}} \ldots \sum_{m_{N_{\mathrm{T}}}=1}^{M_{1 \mathrm{D}}} a\left(\hat{m}_{1}, \ldots, \hat{m}_{N_{\mathrm{T}}-r}, m_{N_{\mathrm{T}}-r+1}, \ldots, m_{N_{\mathrm{T}}}\right)}
$$

as follows

$$
\begin{aligned}
\hat{m}_{1}, \ldots, \hat{m}_{N_{\mathrm{T}}-r} & \left(\frac{1}{2}\|\boldsymbol{y}-\boldsymbol{H} \boldsymbol{s}\|^{2}-\log \left(p\left(m_{1}, \ldots, m_{N_{\mathrm{T}}}\right)\right)\right) \\
=\underset{m_{1}, \ldots, m_{N_{\mathrm{T}}-r}}{\arg \min }(11) & \left(\frac{1}{2}\|\boldsymbol{y}-\overline{\boldsymbol{H}} \overline{\boldsymbol{s}}-\tilde{\boldsymbol{H}} \tilde{\boldsymbol{s}}\|^{2}\right. \\
= & \underset{m_{1}, \ldots, m_{N_{\mathrm{T}}-r}}{\arg \min }\left(-\log \left(p\left(m_{1}, \ldots, m_{N_{\mathrm{T}}}\right)\right)\right) \\
=\underset{m_{1}, \ldots, m_{N_{\mathrm{T}}-r}}{\arg \min } & \left(\frac{1}{2}\left\|\boldsymbol{Q}^{T}(\boldsymbol{y}-\tilde{\boldsymbol{H}} \tilde{\boldsymbol{s}})-\boldsymbol{R} \overline{\boldsymbol{s}}\right\|^{2}\right. \\
& \left.-\sum_{k=1}^{N_{\mathrm{T}}-r} \log \left(p\left(m_{k} \mid m_{k+1}, \ldots, m_{N_{\mathrm{T}}}\right)\right)\right) \\
=\underset{m_{1}, \ldots, m_{N_{\mathrm{T}}-r}}{\arg \min }\left(\frac{1}{2}\|\overline{\boldsymbol{y}}-\overline{\boldsymbol{R}} \overline{\boldsymbol{s}}\|^{2}\right. & \left.-\sum_{k=1}^{N_{\mathrm{T}}-r} \log \left(p\left(m_{k} \mid m_{k+1}, \ldots, m_{N_{\mathrm{T}}}\right)\right)\right) .
\end{aligned}
$$

The first term on the right hand side in (11) is obtained by using the fact that the channel noise is Gaussian. In order to obtain (12), we decompose $\boldsymbol{H}=\left[\begin{array}{ll}\overline{\boldsymbol{H}} & \tilde{\boldsymbol{H}}\end{array}\right]$, $\boldsymbol{s}=\left[\begin{array}{cc}\overline{\boldsymbol{s}}^{T} & \tilde{\boldsymbol{s}}^{T}\end{array}\right]^{T}$, with $\overline{\boldsymbol{H}} \in \mathbb{R}^{N_{\mathrm{R}} \times N_{\mathrm{T}}-r}, \tilde{\boldsymbol{H}} \in \mathbb{R}^{N_{\mathrm{R}} \times r}$, $\overline{\boldsymbol{s}} \in \mathbb{R}^{N_{\mathrm{T}}-r}$, and $\tilde{\boldsymbol{s}} \in \mathbb{R}^{r}$. The expression (13) is obtained by QR-decomposing $\overline{\boldsymbol{H}}=\boldsymbol{Q R}$, where $\boldsymbol{Q} \in \mathbb{R}^{N_{\mathrm{R}} \times N_{\mathrm{R}}}$, and $\boldsymbol{R} \in \mathbb{R}^{N_{\mathrm{R}} \times N_{\mathrm{T}}-r}$, and rewriting $p\left(m_{1}, \ldots, m_{N_{\mathrm{T}}-r}\right)$ in terms of a product of conditional probabilities. Finally, we simplify notation by introducing $\boldsymbol{y}=\left[\begin{array}{cc}\overline{\boldsymbol{y}}^{T} & \tilde{\boldsymbol{y}}^{T}\end{array}\right]^{T}, \boldsymbol{R}=$ $\left[\begin{array}{cc}\overline{\boldsymbol{R}}^{T} & \tilde{\boldsymbol{R}}^{T}\end{array}\right]^{T}$, where $\overline{\boldsymbol{y}} \in \mathbb{R}^{N_{\mathrm{T}}-r}, \tilde{\boldsymbol{y}} \in \mathbb{R}^{N_{\mathrm{R}}-N_{\mathrm{T}}+r}$. $\overline{\bar{R}} \in \mathbb{R}^{N_{\mathrm{T}}-r \times N_{\mathrm{T}}-r}$, and $\tilde{\boldsymbol{R}} \in \mathbb{R}^{N_{\mathrm{R}}-N_{\mathrm{T}}+r \times N_{\mathrm{T}}-r}$, and where we for simplicity assume that $N_{\mathrm{R}} \geq N_{\mathrm{T}}-r$, and arrive at the expression (14).

The proposed approximation of ZF-DF-type to be described supplies an estimate $\hat{m}_{k}^{\mathrm{DF}}$ of $m_{k}$ at a time, for $k=1, \ldots, N_{\mathrm{T}}-$ $r$, starting with $m_{N_{\mathrm{T}}-r}$ and proceeding by decrementing $k$ to 1 . For each $k$, the previous estimates are taken into account. More precisely, these estimates are given by

$$
\begin{aligned}
& \hat{m}_{k}^{\mathrm{DF}}=\underset{m_{k}}{\arg \min }( \\
& \quad \frac{1}{2}\left(\bar{y}_{k}-\bar{R}_{k, k} s_{k}\left(m_{k}\right)-\sum_{i=k+1}^{N_{\mathrm{T}}-r} \bar{R}_{k, i} s_{i}\left(\hat{m}_{i}^{\mathrm{DF}}\right)\right)^{2}
\end{aligned}
$$

$$
\begin{aligned}
& -\log \left(p\left(m_{k} \mid \hat{m}_{k+1}^{\mathrm{DF}}, \ldots, \hat{m}_{N_{\mathrm{T}}-r}^{\mathrm{DF}}, m_{N_{\mathrm{T}}-r+1}, \ldots, m_{N_{\mathrm{T}}}\right)\right) \\
= & \underset{m_{k}}{\arg \min }( \\
& \frac{1}{2}\left(\bar{y}_{k}-\bar{R}_{k, k} s_{k}\left(m_{k}\right)-\sum_{i=k+1}^{N_{\mathrm{T}}-r} \bar{R}_{k, i} s_{i}\left(\hat{m}_{i}^{\mathrm{DF}}\right)\right)^{2} \\
& \left.-\log \left(p\left(m_{k}, \hat{m}_{k+1}^{\mathrm{DF}}, \ldots, \hat{m}_{N_{\mathrm{T}}-r}^{\mathrm{DF}}, m_{N_{\mathrm{T}}-r+1}, \ldots, m_{N_{\mathrm{T}}}\right)\right)\right),
\end{aligned}
$$

for $k=1, \ldots, N_{\mathrm{T}}-r$, where $\bar{R}_{k, i}$ is the element at row $k$ and column $i$ of $\bar{R}^{2}{ }^{2}$ This means that all we need to consider is marginal probabilities. The full ZF-DF scheme is stated in Alg. 1. Finally, we can write our PM estimate as (17) shown on the top of the next page.

$\overline{\text { Algorithm } 1 \text { ZF-DF-type algorithm for approximately solving }}$ (14). The output of the algorithm is $\hat{m}_{1}^{\mathrm{DF}}$ to $\hat{m}_{N_{\mathrm{T}}-r}^{\mathrm{DF}}$.
1: Set $k:=N_{\mathrm{T}}-r+1$.
2: Set $k:=k-1$.
3: Solve (16).
4: If $k>1$, continue from step 2, otherwise terminate.

It is in fact straightforward to use (11) or equivalently (14) as a basis for an algorithm of sphere decoder type, i.e., an algorithm where we are guaranteed to obtain the optimal solution if we have checked all nodes with a smaller distance than the radius, and checked all nodes of one complete branch. This is possible since, for any branch, the marginalized prior probability $p\left(m_{k+1}, \ldots, m_{N_{\mathrm{T}}}\right)$ entering at a sphere decoder node always is less than or equal to the prior probability $p\left(m_{k}, \ldots, m_{N_{\mathrm{T}}}\right)$ entering at the previous node up the branch. This means that the sphere decoder can be used to solve problems involving maximization of joint probabilities including many variables. However, in this paper we stick with Alg. 1, since we focus on parallel implementation with predictable computational complexity. By increasing $r$, MSE performance increases by means of two mechanisms. First, more sums from (6) are retained. Secondly, since $\overline{\boldsymbol{H}}$ has $N_{\mathrm{T}}-r$ columns and $\boldsymbol{H}$ has $N_{\mathrm{T}}$ columns, the condition number of $\overline{\boldsymbol{H}}$ is lower than that of $\boldsymbol{H}$, which improves ZF performance.

In the derivation of Alg. 1, we have left out a sorting of the columns of $\boldsymbol{H}$ and the elements of $s$ for simplicity of the presentation. However, it is a well-known fact, see, e.g., [11], [12], that sorting of the columns of $\boldsymbol{H}$ improves performance. After sorting, the last $r$ columns, over which the summation is performed in (14), should be the columns that

\footnotetext{
${ }^{2} \mathrm{We}$ assume that if $k=N_{\mathrm{T}}-r$, the sums are zero.
} 


$$
\mathrm{E}_{\boldsymbol{x} \mid \boldsymbol{y}}[\boldsymbol{x}] \approx \frac{\sum_{m_{N_{\mathrm{T}}-r+1}=1}^{M_{1 \mathrm{D}}} \cdots \sum_{m_{N_{\mathrm{T}}}=1}^{M_{\mathrm{D}}} \mathrm{E}_{\boldsymbol{x} \mid \hat{m}_{1}^{\mathrm{DF}}, \ldots, \hat{m}_{N_{\mathrm{T}}-r}^{\mathrm{DF}}, m_{N_{\mathrm{T}}-r+1}, \ldots, m_{N_{\mathrm{T}}}}[\boldsymbol{x}] a\left(\hat{m}_{1}^{\mathrm{DF}}, \ldots, \hat{m}_{N_{\mathrm{T}}-r}^{\mathrm{DF}}, m_{N_{\mathrm{T}}-r+1}, \ldots, m_{N_{\mathrm{T}}}\right)}{\sum_{m_{N_{\mathrm{T}}-r+1}=1}^{M_{1 \mathrm{D}}} \cdots \sum_{m_{N_{\mathrm{T}}}=1}^{M_{1 \mathrm{D}}} a\left(\hat{m}_{1}^{\mathrm{DF}}, \ldots, \hat{m}_{N_{\mathrm{T}}-r}^{\mathrm{DF}}, m_{N_{\mathrm{T}}-r+1}, \ldots, m_{N_{\mathrm{T}}}\right)}
$$

would have contributed most to the ZF-DF error if they had been included in the matrix $\overline{\boldsymbol{H}}$ that is involved in the ZFDF process. Moreover, the remaining columns 1 to $N_{\mathrm{T}}-r$ should be sorted in order of decreasing contribution to the ZFDF error. In this way, we minimize error propagation in the ZF-DF algorithm.

We note that the choice of our first approximation in (9) and (10) not is obvious. It may at first sight appear more reasonable to maximize each component of the absolute values of the complete $\mathrm{E}_{\boldsymbol{x} \mid m_{1}, \ldots, m_{N_{\mathrm{T}}}}[\boldsymbol{x}] a\left(m_{1}, \ldots, m_{N_{\mathrm{T}}}\right)$-terms given $m_{N_{\mathrm{T}}-r+1}, \ldots, m_{N_{\mathrm{T}}}$. In preliminary investigations, we also derived an algorithm similar to Alg. 1 but based on maximization of each component of the absolute values of the complete $\mathrm{E}_{\boldsymbol{x} \mid m_{1}, \ldots, m_{N_{\mathrm{T}}}}[\boldsymbol{x}] a\left(m_{1}, \ldots, m_{N_{\mathrm{T}}}\right)$-terms. This algorithm however yielded somewhat lower performance compared to Alg. 1. Alg. 1 is also conceptually simpler and has lower complexity because all vector components are treated simultaneously in the marginalization, while the alternative algorithm has to work on each vector element separately.

The development resulting in (16), Alg. 1, and (17) can be directly generalized to frequency-selective fading channels. We consider a channel

$$
\boldsymbol{y}_{T}=\sum_{t=0}^{N} a(t) \boldsymbol{H}_{t} \boldsymbol{s}_{T-t}
$$

where $\boldsymbol{y}_{T} \in \mathbb{R}^{N_{\mathrm{R}}}$ is the received vector at time $T$, the matrices $\boldsymbol{H}_{t}$ are channels corresponding to different time delays, where the channel power as a function of delay can be adjusted through $a(t) \in \mathbb{R}$, and $s_{t} \in \mathbb{R}^{N_{\mathrm{T}}}$ is the signal vector transmitted in time slot $t$ and corresponding to an i.i.d. source vector $\boldsymbol{x}_{t}$. By setting $\boldsymbol{s}=\left[\boldsymbol{s}_{T}^{T}, \ldots, \boldsymbol{s}_{T+T^{\prime}}^{T}\right]^{T}$, and assuming that $s_{t}=\mathbf{0}$ for $t<T,(16)$ and (17) as well as Alg. 1 can be used directly for frequency-selective fading channels.

\section{Computational COMPLEXity}

We briefly discuss the computational complexity of (17) measured in floating point operations (FLOPS), and start with the pre-processing for each $\boldsymbol{H}$. In the sorting algorithm in [11], [12], the computational complexity of the matrix inversions dominates, and a flat-out computation requires $N_{\mathrm{T}}$ matrix inversions, i.e., a brute force complexity of $N_{\mathrm{T}}^{4}+N_{\mathrm{T}}^{3} N_{\mathrm{R}}$. However, by using the Sherman-Morrison formula, see [18]-[20], the computational complexity can be reduced to $N_{\mathrm{T}}^{3}+N_{\mathrm{T}}^{2} N_{\mathrm{R}}$.

The marginalization of $p\left(m_{1}, \ldots, m_{N_{\mathrm{T}}}\right)$ in order to obtain $p\left(m_{k}, \ldots, m_{N_{\mathrm{T}}}\right)$ for $k=1, \ldots, N_{\mathrm{T}}$, which, because of the sorting of $s$, has to be recalculated for each matrix $\boldsymbol{H}$, i.e., for each fading block, can be efficiently handled, e.g., by representing $p\left(m_{1}, \ldots, m_{N_{\mathrm{T}}}\right)$ in terms of a vector with entries enumerated by assuming that $\hat{m}_{1}$ is the least significant index, and then only summing every $M_{1 \mathrm{D}}$ elements of the vector for obtaining $p\left(m_{2}, \ldots, m_{N_{\mathrm{T}}-r-1}\right)$. Continuing this way, we obtain a complexity estimate of $N_{\mathrm{T}} M_{1 \mathrm{D}}^{N_{\mathrm{T}}}$. This complexity is exponential in $N_{\mathrm{T}}$, but, again, the marginalization only needs to be run once per fading block. We also have the option of not performing pre-sorting of the columns of $\boldsymbol{H}$. For each realization of $\boldsymbol{H}, \mathrm{QR}$ decomposition of $\overline{\boldsymbol{H}}$, as well as calculation of $\boldsymbol{Q}^{T} \tilde{\boldsymbol{H}}$, can be pre-processed.

For each $\boldsymbol{y}$, the evaluation of the expressions $\boldsymbol{Q}^{T} \tilde{\boldsymbol{H}} \tilde{\boldsymbol{s}}$ requires on the order of $r N_{\mathrm{R}} M_{1 \mathrm{D}}^{r}$ FLOPS, $\boldsymbol{Q}^{T} \boldsymbol{y}-\boldsymbol{Q}^{T} \tilde{\boldsymbol{H}} \tilde{\boldsymbol{s}}$ demands around $N_{\mathrm{R}}^{2}+N_{\mathrm{R}} M_{1 \mathrm{D}}^{r}$ FLOPS, and Alg. 1 needs in the order of $\left(\left(N_{\mathrm{T}}-r\right)^{2}+\left(N_{\mathrm{T}}-r\right) M_{1 \mathrm{D}}\right) M_{1 \mathrm{D}}^{r}$ FLOPS. In sufficiently slow fading, the cost of preprocessing each $\boldsymbol{H}$ can be amortized over many vectors $\boldsymbol{y}$. The total number of FLOPS $C_{\boldsymbol{y}}$ needed for the decoding of a realization $\boldsymbol{y}$ is then

$$
C_{\boldsymbol{y}} \approx N_{\mathrm{R}}^{2}+\left(r N_{\mathrm{R}}+\left(N_{\mathrm{T}}-r\right)^{2}+\left(N_{\mathrm{T}}-r\right) M_{1 \mathrm{D}}\right) M_{1 \mathrm{D}}^{r} .
$$

When $N_{\mathrm{T}}$ becomes larger, the complexity of (6), which is proportional to $N_{\mathrm{R}} N_{\mathrm{T}} M_{1 \mathrm{D}}^{N_{\mathrm{T}}}$, will thus always be much larger than (19) if $r$ can be set to a fixed fraction of $N_{\mathrm{T}}$. In the simulations in Sec. IV, we confirm that this is indeed possible.

\section{Simulations}

\section{A. Simulation prerequisites}

The simulation parameters are chosen as follows. We use $M_{1 \mathrm{D}}=2$, i.e., we use binary phase shift keying per real dimension. The source codebook is obtained by the generalized Lloyd algorithm (GLA) [21], which provides a solution that fulfills the the centroid condition (1). For optimization with GLA, we use 10000 realizations of $\boldsymbol{x}$. The elements of $\boldsymbol{H}$ and $\boldsymbol{H}_{t}$ are i.i.d. Gaussian with zero mean and unit variance. Results are calculated as means over channel and source realizations, which are evaluated in the Monte-Carlo sense using 10000 realizations of $\boldsymbol{x}$ and $\boldsymbol{H}$ per CSNR value. The realizations $\boldsymbol{x}$ used for GLA training are strictly different from the realizations used for evaluation. The CSNR is defined as the mean transmitted power divided by the mean noise power, i.e., $\operatorname{CSNR}=\frac{E_{x}\left[\|s\|_{2}^{2}\right]}{N_{\mathrm{R}}}$. We make comparisons to ratedistortion-capacity performance limits calculated using the Gaussian source rate-distortion function, reverse waterfilling, as well as the capacity $\frac{1}{2} \log _{2}\left(\left|\boldsymbol{I}+\frac{E_{\boldsymbol{x}}\left[\|\boldsymbol{s}\|_{2}^{2}\right]}{N_{\mathrm{T}}} \boldsymbol{H}^{T} \boldsymbol{H}\right|\right)$ where $\boldsymbol{I} \in \mathbb{R}^{N_{\mathrm{T}} \times N_{\mathrm{T}}}$ is a diagonal matrix with ones on the diagonal.

\section{B. Results}

Fig. 1 shows a comparison between the proposed method with different $r$, the MMSE solution, the quantization distortion, and the rate-distortion capacity performance limit for $N_{\mathrm{T}}=N_{\mathrm{R}}=8$ and a 1-dimensional zero-mean Gaussian source with variance 1 , in terms of MSE distortion for varying CSNR. Fig. 2 shows the same comparison as in Fig. 1, but for a 2-dimensional zero-mean Gaussian source with covariance matrix $\left[\begin{array}{cc}1 & 0.5 \\ 0.5 & 2\end{array}\right]$. Only the sorting versions of the curves 


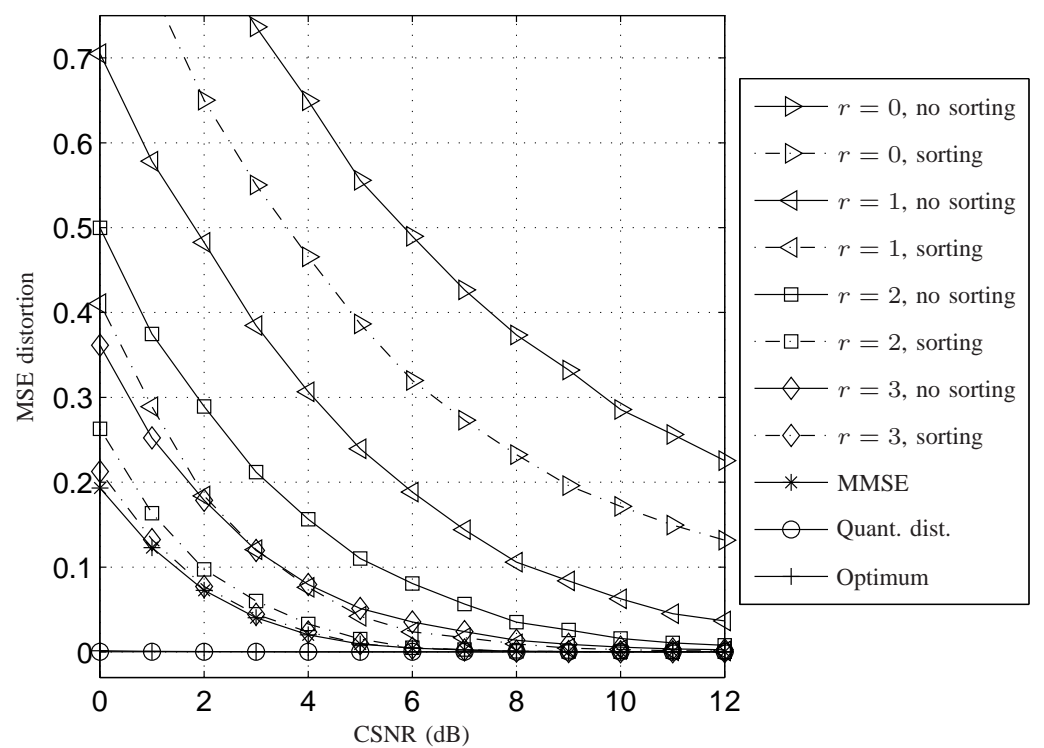

Fig. 1. Comparison of the proposed method with different $r$, sorting and no sorting, the MMSE solution, the quantization distortion, and the rate-distortioncapacity limit, for $N_{\mathrm{T}}=N_{\mathrm{R}}=8$ and a 1-dimensional Gaussian source.

are presented in Fig. 2, and it was verified in separate simulations that sorting improved performance also in this case. Fig. 3 shows the same comparison as in Fig. 1, but for a frequencyselective fading channel with $N_{\mathrm{T}}=N_{\mathrm{R}}=3, T^{\prime}=2$, i.e., source realizations $\boldsymbol{x}_{T}, \ldots, \boldsymbol{x}_{T+T^{\prime}}$ are simultaneously decoded, $\boldsymbol{x}_{t}$ is an i.i.d. 1-dimensional zero-mean Gaussian variable with variance 1 , and $a(0)=1, a(1)=\exp (-1)$.

One observes that with sorting, already for $r=3$, nearMMSE performance is obtained. We conclude from these simulations and investigations of other systems not shown here, that if $N_{\mathrm{T}}=N_{\mathrm{R}}$ and sorting is applied, $r \approx \frac{N_{\mathrm{T}}}{3}$ is sufficient for obtaining near-MMSE behavior for large CSNR regions where the $r=0$-distortion is many times larger than the MMSE distortion. According to Sec. III, the computational complexity of MMSE will be much larger than the PM complexity for larger values of $N_{\mathrm{T}}$ if $r \approx \frac{N_{\mathrm{T}}}{3}$. The gap between the PM method and MMSE seems to diminish when we go to higher CSNR independently of the value of $r$. At high CSNR, we approach the source coding quantization distortion limit. The rate-distortion-capacity limit curves cross the quantization curves at 3.5 and $-4 \mathrm{~dB}$ in Fig. 1 and Fig. 2 respectively. The gap to the rate-distortion-capacity limit results stems from our use of short non-Gaussian codes.

We observe that sorting is important, also in the case of $r=0$. In fact, sorting with $r=2$ gives better performance than $r=3$ without sorting. By increasing $r$, we achieve lower MSE also with the non-sorting approach, and there is thus a tradeoff between using high complexity pre-sorting with low computational complexity for each symbol vector; and not using pre-sorting but instead having high computational complexity for each symbol vector. The simulation code is made available at [22].

\section{CONClusion}

Our setting is analog source transmission over MIMO channels by means of short codes, no transmitter CSI, and full receiver CSI. An approximate fast decoder of PM-type is presented. This new application area of PM algorithms, and the mathematical difficulty of handling prior information that consists of joint probabilities for all symbols in a codeword, are dealt with. In our simulations, the MMSE performance is virtually achieved with around a third of the number of marginalization sums kept. We show that the MMSE algorithm complexity, which is proportional to the number of modulation symbols per antenna to the power of the number of antennas, can be avoided by means of the PM algorithm. We note that pre-processing gives an important reduction in MSE, and there is thus a tradeoff between using high complexity pre-sorting with low computational complexity for each symbol vector; and not using pre-sorting but instead high computational complexity for each symbol vector. Moreover, we show that sphere decoder algorithms can be designed to deal with problems involving general joint prior probabilities.

\section{REFERENCES}

[1] D. Tse and P. Viswanath, Fundamentals of Wireless Communication. Cambridge, U.K.: Cambridge Univ. Press, 2005.

[2] M. Gastpar, B. Rimoldi, and M. Vetterli, "To code, or not to code: lossy source-channel communication revisited," IEEE Transactions on Information Theory, vol. 49, no. 5, pp. 1147 - 1158, May 2003.

[3] J. Karlsson and M. Skoglund, "Optimized low-delay source-channelrelay mappings," IEEE Transactions on Communications, vol. 58, no. 5, pp. 1397-1404, May 2010.

[4] S. Yao and M. Skoglund, "Analog network coding mappings in Gaussian multiple-access relay channels," IEEE Transactions on Communications, vol. 58, no. 7, pp. $1973-1983$, Jul. 2010.

[5] J. G. Proakis and M. Salehi, Communication systems engineering. Upper Saddle River, NJ: Prentice-Hall, 1994.

[6] N. Farvardin, "A study of vector quantization for noisy channels," IEEE Transactions on Information Theory, vol. 36, no. 4, pp. 799-809, Jul. 1990. 


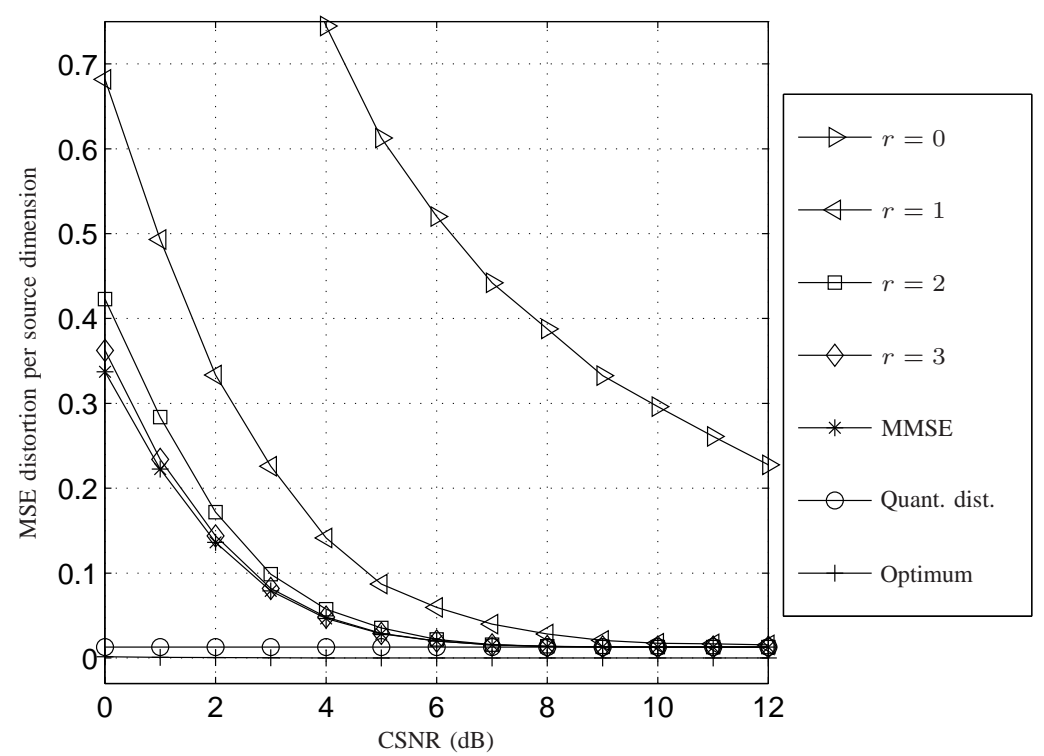

Fig. 2. This figure shows the same comparison as in Fig. 1, with sorting for a 2-dimensional Gaussian source.

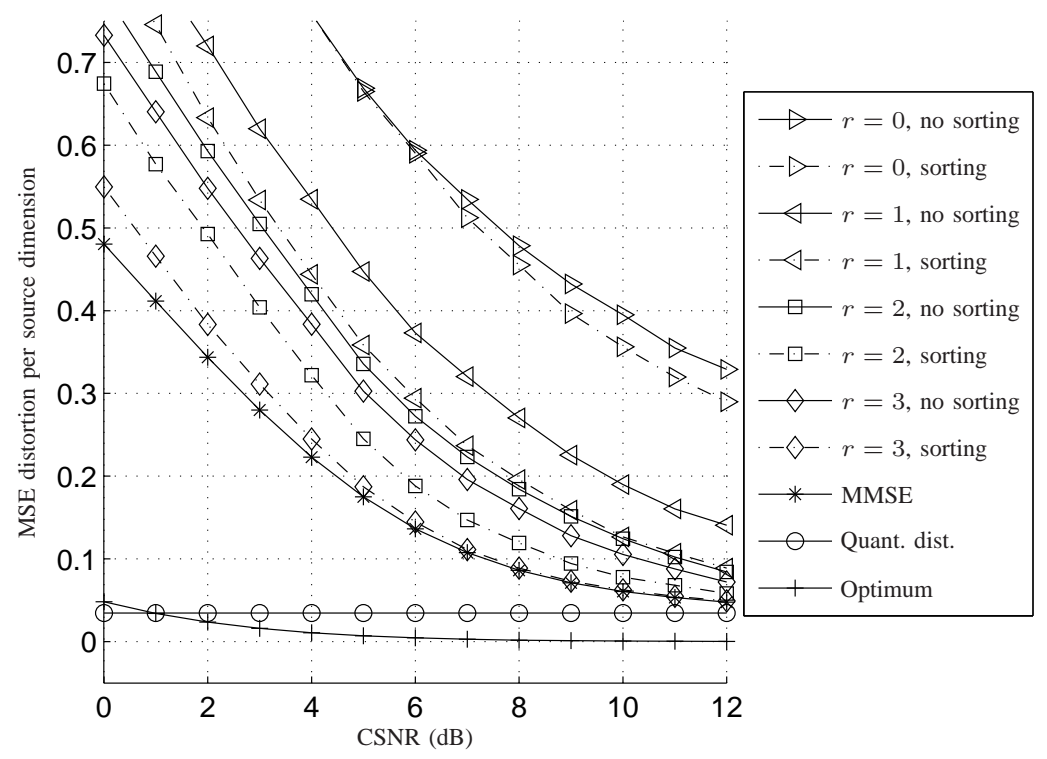

Fig. 3. This figure shows the same comparison as in Fig. 1, but for a frequency-selective fading channel with $N_{\mathrm{T}}=N_{\mathrm{R}}=3, T^{\prime}=2$, i.e., three sources $\boldsymbol{x}_{T}, \ldots, \boldsymbol{x}_{T+T^{\prime}}$ are simultaneously decoded, $a(0)=1, a(1)=\exp -1$, and $\boldsymbol{x}_{t} \in \mathbb{R}$ being zero-mean Gaussian.

[7] D. Persson and T. Eriksson, "Power series quantization for noisy channels," IEEE Transactions on Communications, vol. 58, no. 5, pp. $1405-1414$, May 2010.

[8] — " "On multiple description coding of sources with memory," vol. 58, no. 8 , pp. $2242-2251$, Aug. 2010.

[9] D. Persson, J. Kron, M. Skoglund, and E. G. Larsson, "Joint sourcechannel coding for the MIMO broadcast channel," IEEE Transactions on Signal Processing, vol. 60, no. 4, pp. 2085 -2090, Apr. 2012.

[10] M. Skoglund and P. Hedelin, "Hadamard-based soft decoding for vector quantization over noisy channels," IEEE Transactions on Information Theory, vol. 45, no. 2, pp. 515 -532, Mar. 1999.

[11] E. G. Larsson and J. Jaldén, "Fixed-complexity soft MIMO detection via partial marginalization," IEEE Transactions on Signal Processing, vol. 56, no. 8, pp. 3397-3407, Aug. 2008.

[12] D. Persson and E. G. Larsson, "Partial marginalization soft MIMO detection with higher order constellations," IEEE Transactions on Signal Processing, vol. 59, no. 1, pp. 453 -458, Jan. 2011.

[13] L. Barbero and J. Thompson, "Fixing the complexity of the sphere decoder for MIMO detection," IEEE Transactions on Wireless Communications, vol. 7, no. 6, pp. 2131-2142, June 2008.

[14] _ _ "Extending a fixed-complexity sphere decoder to obtain likelihood information for turbo-MIMO systems," IEEE Transactions on Veh. Technol., vol. 57, no. 5, pp. 2804-2814, Sept. 2008.

[15] M. Čirkić, D. Persson, and E. G. Larsson, "Allocation of Computational Resources for Soft MIMO Detection," IEEE Journal of Selected Topics in Signal Processing, vol. 5, no. 8, pp. 1451-1461, 2011.

[16] M. Črkić, D. Persson, E. G. Larsson, and J.-A. Larsson, "Gaussian approximation of the LLR distribution for the ML and partial marginalization MIMO detectors," in Proc. ICASSP, 2011.

[17] E. Agrell, T. Eriksson, A. Vardy, and K. Zeger, "Closest point search in lattices," IEEE Transactions on Information Theory, vol. 48, no. 8, pp. 2201-2214, Aug 2002.

[18] J. Benesty, Y. Huang, and J. Chen, "A fast recursive algorithm for optimum sequential signal detection in a BLAST system," IEEE Transactions on Signal Processing, vol. 51, no. 7, pp. 1722 - 1730, Jul. 2003.

[19] T.-H. Liu and Y.-L. Liu, "Modified fast recursive algorithm for efficient 
MMSE-SIC detection of the V-BLAST system," IEEE Transactions on Wireless Communications, vol. 7, no. 10, pp. 3713 -3717, Oct. 2008.

[20] T.-H. Liu, "Some results for the fast MMSE-SIC detection in spatially multiplexed MIMO systems," IEEE Transactions on Wireless Communications, vol. 8, no. 11, pp. 5443 -5448, Nov. 2009.

[21] Y. Linde, A. Buzo, and R. Gray, "An algorithm for vector quantizer design," IEEE Transactions on Communications, vol. 28, no. 1, pp. 84 - 95, Jan. 1980.

[22] Publications at the Communication Systems Division, Linköping University. [Online]. Available: http://www.commsys.isy.liu.se/en/publications 